\title{
Researches on Flow Field's Dynamic Features of Vacuum Switching Arc Based on Optical Flow Field
}

\author{
Huajun Dong ${ }^{1,}$, Chunxiao $\mathrm{Li}^{1}$, Yingjie Guo ${ }^{1,2}$ and $\mathrm{Jie}_{\mathrm{Li}}{ }^{1}$ \\ ${ }^{1}$ School of Mechanical Engineering, Dalian Jiaotong University, Dalian, China \\ ${ }^{2}$ State Grid Tianjin Pinggao Intelligent Electirc Co., Ltd., Tianjin, China \\ ${ }^{*}$ Corresponding author
}

\begin{abstract}
As a kind of fluid, the motion of vacuum arc is complicated and various. Its structure and distribution have a significant effect on vacuum interrupters' comprehensive properties. A method to analyze of arc flow field's dynamic feature based on optical flow is proposed aiming at complicated flow field of vacuum switch arc's sequence images. In this paper optical flow field's basic principle is firstly introduced, and then the noise filtering method to process arc images in different stages is used. Finally, the method of optical flow field estimation to obtain pictures of arc flow field's motion vector feature in the vacuum gap is adopted. According to the results we can find that in the stage of arc striking, the pressure of metal vapor in the vacuum gap is low, accompanied by an expansion of arc and the formation of several parts of eddy current zones. When the arc is going to be extinguished, with the decreasing of the temperature of vacuum arc column center, the arc plasma converges upon its column center instead of expanding, and the parts of eddy current zone shrinks and disappears. At the same time we can see that the method of optical flow field can obtain vacuum arc optical flow field's dynamic feature effectively to describe arc particle moving direction and size. Target images can also be divided easily, real-timely, and accurately, which provides a new technical mean to analyze the dynamic feature of vacuum arc flow field.
\end{abstract}

Keywords—vacuum arc; arc flow field; optical flow field; eddy current

\section{INTRODUCTION}

As one of the equipments to control and protect power system, high voltage circuit breakers play an important role in safe system running [1, 2]. Vacuum switches, which contain advantages of small volume, long life, and excellent breaking capability, have been widely applied in the field of medium voltage. Furthermore, their application in high voltage will also be widely accepted [3-5]. Nowadays, some bottleneck problems of basic theory of vacuum arc still need to be studied, containing the lack of arc flow field dynamic feature theory, which restricts vacuum switches' development and application. As a kind of fluid, vacuum arc exists in vacuum interrupter as metal vapor, which is full of complex features, but the rule of its motion is hard to express in simple mathematical type. One of the most important things to research on vacuum switch arc basic theory is to do quantitative analysis in arc flow field features. Therefore, it is with great significance to research on vacuum switching arc flow field dynamic features deeply.

Flow field features are detected and analyzed by three methods: topology analysis, physical analysis, and image processing [6]. Li Xiaocong extracted ocean eddy features, and proposed a location mode based on sperner lemma from combinatorial topology, which made eddies more visual [7]; $\mathrm{Xu}$ Huaxun proposed a method to intelligently extract typical flow field features based on BP neural network [8]; Dong Huajun successfully drew distribution map of vacuum arc burning 2D speed field using PIV technology, which provided technical support on arc flow field features researches [9]. In recent years, with the development of computer technology and digital image processing, the calculation method of optical flow based on image sequence has been widely applied. One of the most important applications is to use image sequences to extract flow field features, and realize the analysis of its dynamic features.

Optical Flow Field calculation is a method to research on the relationship between image gray scale changing with time and object motion. Optical flow is instantaneous speed vector of pixel, and optical flow field is vector field. The advantage of this method is that it can detect moving object independently without background restriction. Nowadays, optical flow method has been applied widely in detecting and tracking moving objects, but few apply in arc flow field detection. In this paper, the method of MATLAB optical flow combined with arc images collected by CMOS high speed camera is proposed to analyze vacuum arc flow field during striking and distinguishing stage. It also described appearance and harms of eddy current zone, which provide technology support on quantitative analysis of arc dynamic features.

\section{OPTICAL FLOW ESTIMATION BASIC PRINCIPLE}

\section{A. Optical Flow Estimation Basic Equation}

Assuming that gray value of pixel point $(\mathrm{x}, \mathrm{y})$ at time $t$ is $I(x, y, t)$, and this point at time $t+\Delta t$ changes into $(x+\Delta x, y+\Delta y)$, and its gray value is $I(x+\Delta x, y+\Delta y, t+\Delta t)$. According to gray consistency hypothesis $\mathrm{d} I(x, y, t) / \mathrm{d} t=0$, which means the gray value of this point doesn't depend on its motion. Then

$$
I(x, y, t)=I(x+\Delta x, y+\Delta y, t+\Delta t)
$$

Expend (1) with Taylor Formula, then

$$
I(x+\Delta x, y+\Delta y, t+\Delta t)=I(x, y, t)+\frac{\partial I}{\partial x} \Delta x+\frac{\partial I}{\partial y} \Delta y+\frac{\partial I}{\partial t} \Delta t+\varepsilon
$$



then

In (2), second order infinitesimal $\varepsilon$ is neglected, $\Delta t \rightarrow 0$,

$$
\begin{gathered}
\frac{\partial I}{\partial x} \frac{\mathrm{d} x}{\mathrm{~d} t}+\frac{\partial I}{\partial y} \frac{\mathrm{d} y}{\mathrm{~d} t}+\frac{\partial I}{\partial t}=0 \\
\operatorname{In}(3), \boldsymbol{u}=\mathrm{d} x / \mathrm{d} t, \boldsymbol{v}=\mathrm{d} y / \mathrm{d} t, \text { then } \\
I_{x} \boldsymbol{u}+I_{y} \boldsymbol{v}+I_{t}=0
\end{gathered}
$$

Optical flow estimation basic equation is as (4), and it can also be expressed into vector:

$$
\nabla I \cdot w+I_{t}=0
$$

In $(5), \nabla I=\left(I_{x}, I_{y}\right)^{T}$ represents direction of spatial gradient, and $\boldsymbol{w}=(\boldsymbol{u}, \boldsymbol{v})^{T}$ represents optical flow vector.

However, two variables of optical Flow, $\boldsymbol{u}$ and $\boldsymbol{v}$, cannot be determined uniquely by only one equation. As a consequence, in order to solve this ill-posed problem, a method called HornSchunck algorithm was proposed by American scholar B.K.P.Horn and B.G.Schunck [10].

\section{B. Horn-Schunck Algorithm}

In order to calculate optical flow, global smoothness constraint needs to be introduced, which means smoothness and optical estimation basic equation mentioned above require being satisfied. The errors generated from two parts, $E_{\text {Smooth }}$ and $E_{\text {Data, }}$, are presented as (6) and (7).

$$
\begin{gathered}
E_{\text {Smooth }}=\min \left\{\left(\frac{\partial \boldsymbol{u}}{\partial x}\right)^{2}+\left(\frac{\partial \boldsymbol{u}}{\partial y}\right)^{2}+\left(\frac{\partial \boldsymbol{v}}{\partial x}\right)^{2}+\left(\frac{\partial \boldsymbol{v}}{\partial y}\right)^{2}\right\} \\
E_{\text {Data }}=\min \left\{I_{x} \boldsymbol{u}+I_{y} \boldsymbol{v}+I_{t}\right\}
\end{gathered}
$$

The two equations represent that both the value of $|\nabla \boldsymbol{u}|^{2}+|\nabla \boldsymbol{v}|^{2}$ in fixed neighborhood and the error that optical flow field deviates from basic equation require to be as small as possible. Then, optical estimation formula calculated by Horn-Schunck algorithm is obtained by (6) and (7), as in (8).

$$
\begin{gathered}
E=\min \left\{\int \int \alpha ^ { 2 } \left[\left(\frac{\partial u}{\partial x}\right)^{2}+\left(\frac{\partial u}{\partial y}\right)^{2}+\left(\frac{\partial v}{\partial x}\right)^{2}+\left(\frac{\partial v}{\partial y}\right)^{2}+\right.\right. \\
\left.\left.\left(I_{x} \boldsymbol{u}+I_{y} v+I_{t}\right)^{2}\right] \mathrm{~d} x \mathrm{~d} y\right\}
\end{gathered}
$$

Where $\alpha$ is coefficient factor of $E_{\text {Smooth. }}$ The larger the value of $\alpha$ is, the better the effect of Smoothness is.

Through the transformation of the variational problem presented as (8), corresponding Euler Equation can be obtained, and then the speed vectors of each pixel in the image $\left(u^{n+1}, v^{n+1}\right)$ through $(n+1)$ iteration, solved by Gauss-Seidel, are presented as (9).

$$
\begin{aligned}
& \boldsymbol{u}^{(n+1)}=\overline{\boldsymbol{u}^{(n)}}-I_{x} \cdot \frac{I_{x} \overline{\boldsymbol{u}^{(n)}}+I_{y} \overline{\boldsymbol{v}^{(n)}}+I_{t}}{\alpha^{2}+I_{x}^{2}+I_{y}^{2}} \\
& \boldsymbol{v}^{(n+1)}=\overline{\boldsymbol{v}^{(n)}}-I_{y} \cdot \frac{I_{x} \overline{\boldsymbol{u}^{(n)}}+I_{y} \overline{\boldsymbol{v}^{(n)}}+I_{t}}{\alpha^{2}+I_{x}^{2}+I_{y}^{2}}
\end{aligned}
$$

Where the number of iteration $n$ is related to the initial value of optical flow. Generally, assume that the initial value is zero, which means $\boldsymbol{u}^{(0)}=\boldsymbol{v}^{(0)}=0$. Iteration will finish when the difference of iteration between before and after is less than a given value.

\section{ARC IMAGES ACQUISITION AND PREPROCESSING}

\section{A. Arc Images Acquisition}

According to the research of Schade E. as [11], vacuum switches interrupting process is divided into four stages: striking, burning, extinguishing, and post-arc dielectric recovery. Collect vacuum arc images in different stages using CMOS high speed camera. Figure (1) shows the system of arc images acquisition, which contains vacuum switches generating device, optical system, data acquisition system, computer processing equipment, and system of diagnosing plasma temperature and arc shape [12]. Photography unit is one of the most important parts, and image sensor is its core component. First, it changes optical signal into electrical signal, than input this electrical signal into computer through $\mathrm{A} / \mathrm{D}$ conversion interface. At present, mainstream image sensor is CCD and CMOS. CCD camera grabs images more slowly, but the image quality is better, thus it costs more. With the development of photography technology, CMOS camera grabs images quickly, and the quality has been increased significantly As a consequence, in order to research on arc flow field dynamic features, CMOS camera can grab more high quality images in finite time, which can detect flow field changing more accurately.

\section{B. Arc Images Preprocessing}

In the process of image acquisition, considering the environment around, noises cannot be avoided in images, which decrease images quality. In order to analyze arc flow field features more accurately, it is essential to filter noises before calculate optical flow filed. In this paper, gray-scale transformation algorithm is chosen to filter noises. Adjust the gray value into a fixed range by histogram, which has good performance in contrast enhancing and noise filtering.

First, select two adjacent frames arc images in the stage of arc striking, and adjust the gray value into a fixed range, as shown in Figure (2)Then select two adjacent frames arc images in the stage of arc extinguishing, using the same method as striking stage, as shown in Figure (3). 


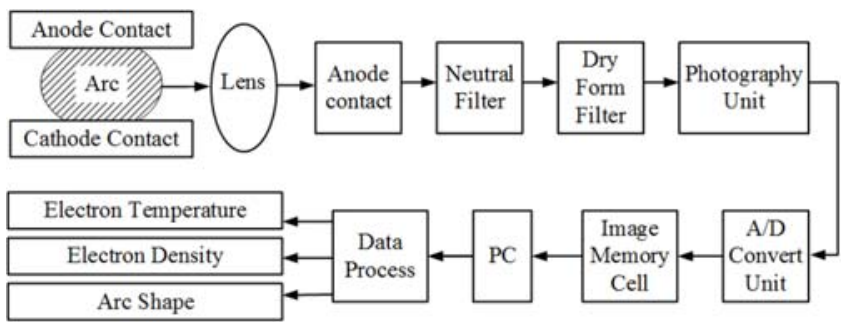

FIGURE I. ACQUISITION SYSTEM OF VSA IMAGES
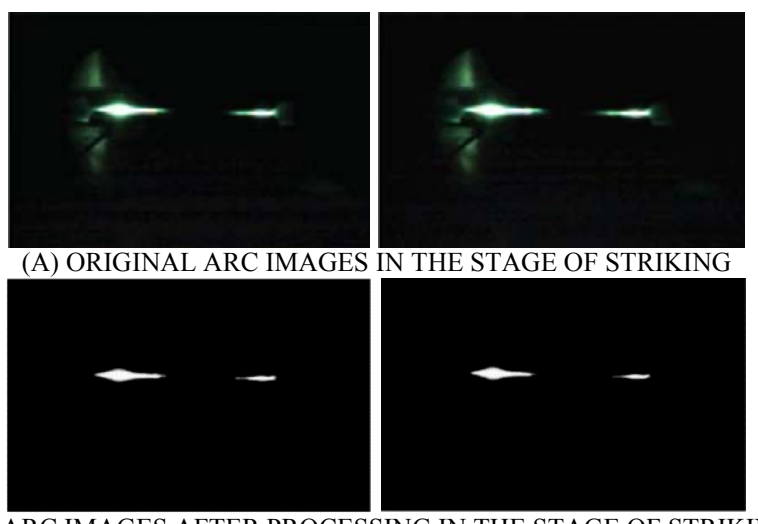

(B) ARC IMAGES AFTER PROCESSING IN THE STAGE OF STRIKING

FIGURE II. NOISE FILTERING RESULT BETWEEN TWO ADJACENT FRAMES ARC IMAGES IN THE STAGE OF ARC STRIKING
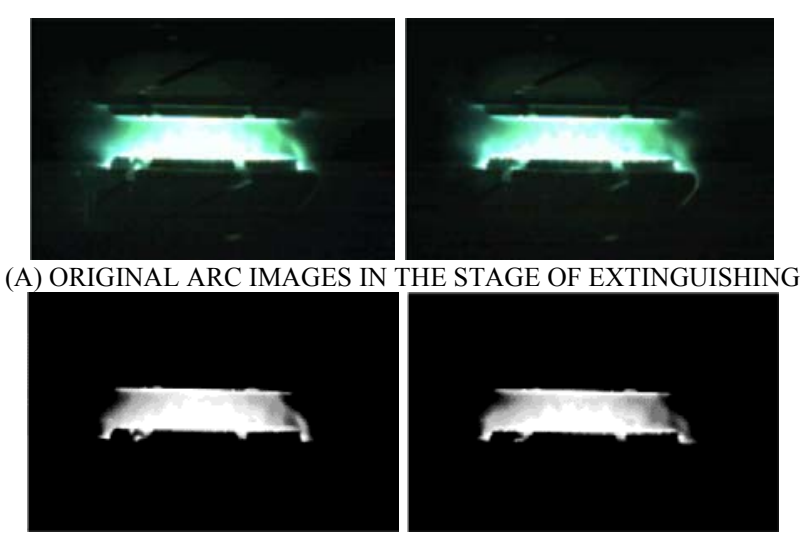

(B) ARC IMAGES AFTER PROCESSING IN THE STAGE OF EXTINGUISHING

FIGURE III. NOISE FILTERING RESULT BETWEEN TWO ADJACENT FRAMES ARC IMAGES IN THE STAGE OF ARC EXTINGUISHING

\section{RESUlT ANALYSIS}

\section{A. Arc Characters and Flow Field Dynamic Features} Analysis in the Stage of Striking

The instant the vacuum switch contacts separate, arc plasma converge quickly and current concentrates on several points to make temperature increase rapidly. Due to this reason, metal vapor is generated from electrode surface, and furthermore, arc generates in vacuum gap. The stage of arc striking has influence on arc expending and extinguishing, therefore, the study of this stage is of great significance. In this paper, the software MATLAB is used to analyze arc images in arc striking stage, then obtain optical flow field of two adjacent frames arc images, as shown in Figure (4). In the pictures, background is the first frame image, and the direction and length of arrows represent the direction and value of instantaneous velocity.

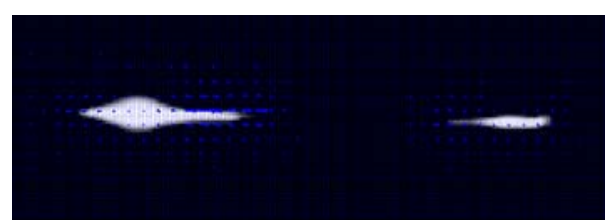

(A) ARC OPTICAL FLOW FIELD IN THE STAGE OF STRIKING

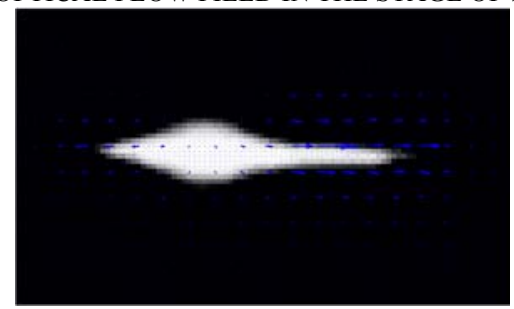

(B) PARTIAL ARC OPTICAL FLOW FIELD IN THE STAGE OF STRIKING

FIGURE IV. THE OPTICAL FLOW FIELD BETWEEN TWO ADJACENT FRAMES ARC IMAGES IN THE STAGE OF ARC STRIKING

From Figure (4), in the stage of arc striking, the distance between electrodes is small, metal vapor is generated in vacuum gap, and bridge column arc appears between two contacts. Meanwhile, current flows through metal bridge column and increased rapidly, accompanied by arc plasma spreading around. With the distance between contacts further expansion, metal bridge was longer, more metal vapor was generated, causing field emission, which lead to the formation of cathode spot. However, in this moment, metal vapor has low pressure gradient, so the spreading speed of column center is slower than surrounding.

\section{B. Arc Characters and Flow Field Dynamic Features \\ Analysis in the Stage of Extinguishing}

In the stage of extinguishing, arc characters decide the area of melting, where metal vapor can be released after current zero, which causes arc restriking. Therefore, arc extinguishing stage is the key point that vacuum switches can interrupt successfully, which is also the main work to research vacuum circuit breaker under high current [13]. Optical flow field of two adjacent frames arc images in arc extinguishing stage is as shown in Figure (5).

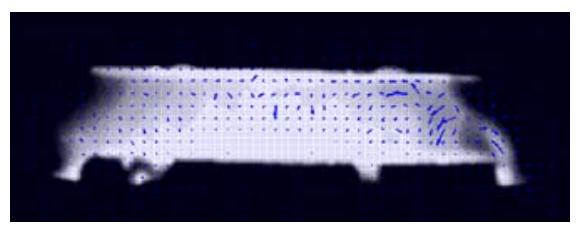

(A) ARC OPTICAL FLOW FIELD IN THE STAGE OF EXTINGUISHING 


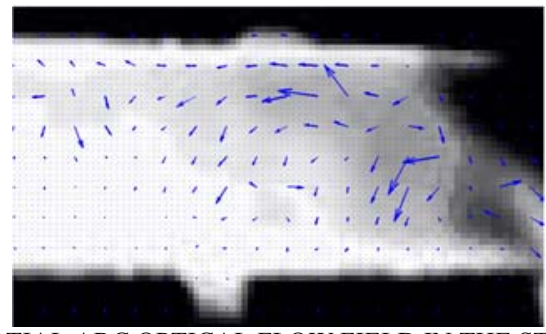

(B) PARTIAL ARC OPTICAL FLOW FIELD IN THE STAGE OF EXTINGUISHING

\section{FIGURE V. THE OPTICAL FLOW FIELD BETWEEN TWO ADJACENT FRAMES ARC IMAGES IN THE STAGE OF ARC EXTINGUISHING}

In Figure (5), in the arc extinguishing stage, with the increasing distance of contacts expansion, arc current and power is decreasing, which causes metal vapor pressure and the temperature in vacuum gap get lower. At the moment, the arc plasma converges upon its column center instead of expanding. Considering the influence of magnetic field, eddy current zone appears in axial magnetic field. With the current get lower, magnetic field force decrease, accompanied by the eddy current zone shrinks and disappears. However, the magnetic generated by eddy current is slower than current, which will cause harmful effect on the rest plasma. In order to avoid arc restrikes after current zero and enhance breaking capability of vacuum interrupter, magnetic induction from eddy current needs to be decreased.

\section{CONCLUSIONS}

In this paper, Horn-Schunck algorithm was applied in the analysis of vacuum switch arc flow field features. First, the optical flow field of two adjacent frames arc images was obtained, and then used MATLAB to analyze the features. According to the results, the main conclusions in this paper have been summarized as follows:

(1) Using Horn-Schunck algorithm, arc flow field and eddy current dynamic features can be analyzed efficiently and the effect is good.

(2) In the stage of arc striking, arc plasma spreads from column center, where the pressure gradient of metal vapor in column center is lower. As a consequence, the speed of arc spreading in the column center is slower than it around. In the stage of arc extinguishing, metal vapor pressure gets decreased, and the temperature in column center is lower, so the motion direction of arc plasma converges upon its column center. Besides, eddy current inhibit the expending of arc. With the arc extinguishing, magnetic field intensity decreases, and eddy current zone get smaller and disappear.

(3) The generation of eddy current will decrease the capability of post-arc dielectric recovery, which do harm to vacuum interrupter breaking. So it is essential to inhibit the magnet field generated by eddy current.

\section{ACKNOWLEDGMENT}

This work was financially supported by China Natural Scientific Fundamental Research Foundation under Award
(No.51477023, No.51207016) and Educational Commission of Liaoning Province of China (No.JDL2017032).

\section{REFERENCES}

[1] Yang, L.X., and Zhu Y.L. "High voltage circuit breaker fault diagnosis of probabilistic neural network," Power System Protection and Control,vol.43:10, 2015, pp. 62-67.

[2] Zhang P., Zhao S.T., Shen L., and Zhao X.P. "Research on vibration and acoustic joint mechanical fault diagnosis method of high voltage circuit breaker based on improved EEMD," Power System Protection and Control, vol.42:8, 2014, pp. 77-81.

[3] Liu B. and Wu J.W. "Morphology of forcing interruption arc of DC vacuum arc," Transactions of China Electrotechnical Society, vol.31:24, 2016, pp. 157-163.

[4] Zhou Y. and Wang B.H. "Mechanical characteristic state evaluation of vacuum circuit breaker based on multi-sensor signal fusion," Electrical Engineering, vol.17:6, 2016, pp. 30-35.

[5] Liu Z.Y., Wang J.M., Xiu S.X., et al. "Development of High-Voltage Vacuum Circuit Breakers in China," Plasma Science IEEE Transactions, vol.35:4, 2007, pp. 856-865.

[6] Post, F. H., Vrolijk, B., Hauser, H., Laramee, R. S., and Doleisch, H. "The state of the art in flow visualisation: feature extraction and tracking," Computer Graphics Forum, vol.22:4, 2003, pp. 775-792.

[7] Li X.C. "Feature extraction of ocean field vortices and visualization research," Qingdao, Ocean University of China, 2009.

[8] Xu H.X. "Extraction and visualization for complexflow field features," Changsha, National University of Defense Technology, 2011.

[9] Dong H.J., Kang K., Zang K., Guo F.Z, and Zou J.Y. "Particle-ImagingVelocimetry study of flow field of vacuum switching arc in short gap," Journal of Materials Processing Technology, vol.34:6, 2014, pp. 590-592.

[10] Berthold K.P. Horn and Brian G. Schunck. "Determining optical flow," Artificial Intelligence, vol.17:1, 1981, pp. 185-203.

[11] Schade E. "Physics of high-current interruption of vacuum circuit breakers," IEEE Transactions on Plasma Science, vol.33:5, 2005, pp. 1564-1575.

[12] Dong H.J., Liao M.F., Zou J.Y., Qiu H.H., and Zhou Z.Y. "Collection and processing procedure of vacuum switches arc images," Transactions of China Electrotechnical Society, vol.22:8, 2007, pp. 174-177.

[13] Wang L.J., Hu L.L., Zhou X., et al. "Simulation of high-current vacuum arc characteristics with big-size electrode conditions," Transactions of China Electrotechnical Society, vol.28:2, 2013, pp. 163-170. 